\title{
FEATURE
}

\section{The changing face of HIV in China}

\section{HIV has advanced from high-risk groups such as intravenous drug users to some in the general population, according to comprehensive new data from the south of China. What needs to be done to halt its spread?}

\section{Lin Lu, Manhong Jia, Yanling Ma, Li Yang Zhiwei Chen, David D. Ho, Yan Jiang and Linqi Zhang}

The HIV-1/AIDS epidemic in China is at a critical juncture. Historically, HIV-1 infection has been largely confined to certain high-risk populations such as intravenous drug users and former blood and plasma donors in geographically disparate rural areas $^{1-3}$. However, HIV-1 prevalence has now increased rapidly among men who have sex with men and among female sex workers ${ }^{4,5}$. It seems that China is following the path of some of the other Asian countries where HIV-1 infection is no longer confined to high-risk population ${ }^{5}$.

Since the first cases among foreign tourists and local recipients of imported factor VIII in the mid 1980s, HIV-1 has spread to all of mainland China ${ }^{1,4}$. The current epidemic comprises largely of two affected populations: former blood and plasma donors in Henan and neighbouring provinces, and intravenous drug users in Yunnan and along drug-trafficking routes ${ }^{1,2}$ (Fig. 1). Both of these populations stemmed from the infection of drug users from Yunnan's Dai and Jingpo ethnic minority groups in Yunnan in the late $1980 \mathrm{~s}^{6,7}$.

Statistics from the Chinese Ministry of Health and UNAIDS have revealed a worrisome trend of the HIV-1 epidemic in China. As of October 2007, an estimated 700,000 infections had occurred ${ }^{4}$. Although the prevalence of infection remains low $(0.04-0.07 \%)$, the new figure represents an $8 \%$ increase since 2006 (refs 4,5). Remarkably, $38 \%$ of the cases were attributed to heterosexual contacts - more than triple the $11 \%$ in 2005 (ref. 4). In line with this trend, the proportion of women infected has doubled over the past decade ${ }^{4}$. As $90 \%$ of these women are of child-bearing age (15-44), this is likely to translate into more vertical transmission from mother to child ${ }^{4}$. Additionally, the proportion of cases among men who have sex with men increased eight-fold from $0.4 \%$ in 2005 to $3.3 \%$ in 2007 (ref. 4). These data suggest that the HIV1 epidemic is expanding, and that more effective preventive measures are urgently needed.

\section{The epidemic in Yunnan}

Located in southwest China, Yunnan has long been regarded as China's Shangri-la for its natural beauty. But now, with all of its 16 prefectures affected, it is a major site of

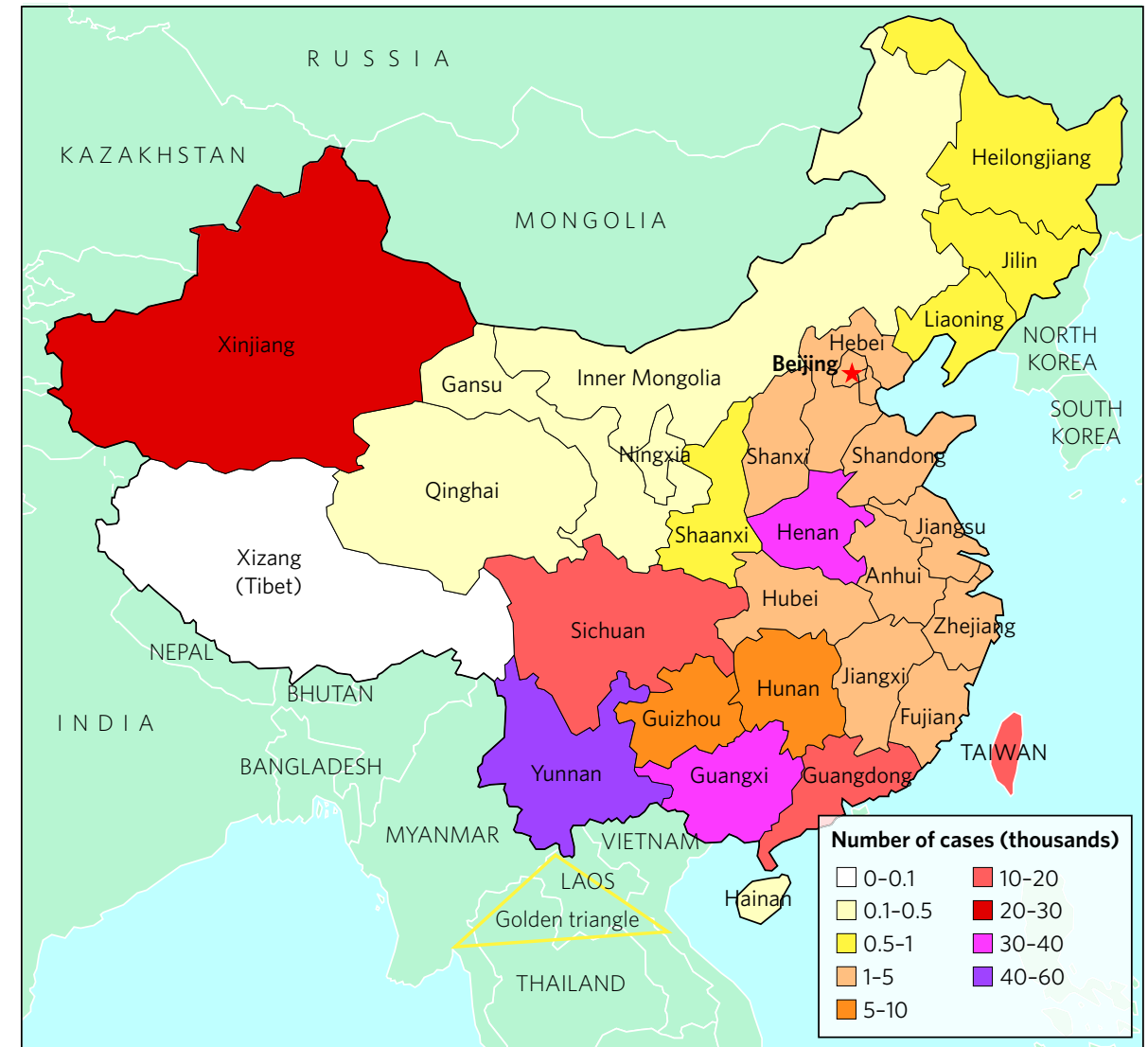

Figure 1 | Pervasive spread. The geographic distribution of cumulative reported HIV-1 infection in mainland China (source: ref. 4).

the AIDS epidemic. Yunnan's ethnic diversity is unrivalled in China, with 25 different ethnic minority groups representing one third of the province's population. Of these groups, 13 live along the border with Myanmar, Laos and Vietnam, and cross-border travel and commerce are common. Yunnan has a long history of opium/heroin trade, and the vast majority of illicit drugs in China are trafficked through Yunnan from the 'Golden triangle' of illicit opium production, encompassing Myanmar, Thailand, Laos and Vietnam (Fig. 1) 8,9 $^{8,}$

HIV-1 was detected in intravenous drug users inYunnan in 1989 (ref. 10). It then also spread among other populations ${ }^{11}$. Between 1989 and 2006, 3.2 million blood samples were tested in Yunnan. This testing identified 48,951 HIV-1 cases, 3,935 AIDS patients, and 1,768 resultant deaths - representing about $25 \%, 8 \%$ and $13 \%$ of the national totals, respectively. Prefectures bordering Myanmar and Vietnam were the first and the most severely affected.

Although the cumulative HIV-1 case load rose gradually from 1989 to 2003 , there was a sharp rise in 2004, when 13,486 new cases were seen. This total is comparable to the number identified in the previous 16 years. Identification of these new cases was likely to be due to increased surveillance and testing since the estimated incidence rates remained relative stable over time among the major risk groups. These estimates were determined by re-testing all seropositive samples from the surveillance effort using the BED assay ${ }^{12}$ to detect those with low-affinity antibodies to HIV-1. Intravenous drug users had the highest incidence rate throughout the study, varying between $2.2 \%$ and $8.0 \%$ per year, whereas that for outpatients attending sexually transmitted infection (STI) clinics was $0.3-1.0 \%$ 
per year and for pregnant women it was about $0.1 \%$ per year.

\section{Changing demographics}

HIV-1 has hit different Yunnan populations disproportionately. Figure 2 shows provincial average HIV-1 prevalence rates over time among intravenous drug users, female sex workers, STI outpatients, pregnant women and an 'unlinked population' (patients admitted to the hospital who were willing to be tested for HIV-1 anonymously) based on the results from 97 sentinel surveillance sites located through the province. Within these groups, the highest prevalence rate has always been found in the intravenous drug users population. From 1992 to 1995, the average prevalence rate remained around $6 \%$. In 1996 , it jumped to about $22 \%$, and then remained near that level. Prevalence rates among female sex workers and STI outpatient groups have been consistently lower, but follow a similar trend. The 'unlinked' and pregnant women populations have also experienced a similar pattern of prevalence increases, although the jumps occurred in 1999 and 2003, respectively. This sequential upsurge of infection among intravenous drug users, female sex workers, STI outpatients, then among the 'unlinked' and finally pregnant women, is reminiscent of what has been seen in other countries, where HIV-1 infection has spread from high-risk groups to some in the general population.

This trend in transmission mode is further illustrated by the fact that the proportion of cases among intravenous drug users has decreased from $100 \%$ in 1989 to $40 \%$ in 2006. Concurrently, heterosexual transmission has increased markedly, reaching $37.5 \%$ of infections in 2006.

Although most infections were in farmers from 1989 to 1995 , more factory workers are now infected, and the number of infections among unemployed persons have come to rival those in farmers. In addition, whereas the Dai and Jingpo minorities were the most affected ethnic groups in 1989-95, Han Chinese overtook these minorities in 1996 and up to 2006 accounted for around $60 \%$ of infections.

Changes in age distribution are also evident. Although on average more than $95 \%$ of infected individuals have been aged 20-40, HIV-1 prevalence has increased among the 30-59 group and decreased among the 20-29 group. This could be attributed to ageing of infected individuals or to new infections of relatively older age groups over time. Nonetheless, high prevalence in the 20-29 and younger than 20 age groups suggests ongoing infection within the young population.

HIV-1 in Yunnan has also spread to the

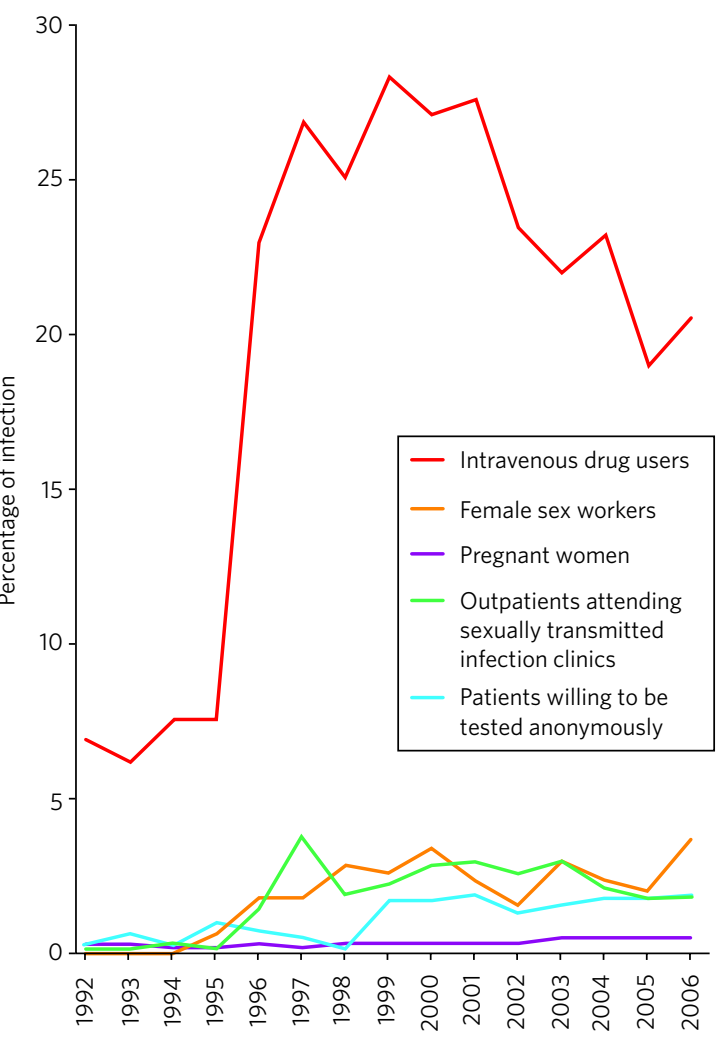

igure 2 Changing trends. HIV-1 prevalence among various risk roups in Yunnan between 1992 and 2006.

female population. Before 1996, most infected individuals were male. However, from 19962006, the proportion of HIV-1-infected women gradually increased from $7.1 \%$ to $35 \%$, and the male to female ratio decreased from 13:1 to $1.9: 1$.

\section{Virus evolution}

With the dramatic changes in disease distribution, HIV-1 genetics in Yunnan have become increasingly complex. The initial HIV-1 epidemic among intravenous drug users in Yunnan in 1989 was caused by a mixture of viruses closely resembling European/North American subtype $B$ and Thai subtype $B\left(B^{\prime}\right)^{13}$. But by 1996 , the B' subtype began to dominate ${ }^{13,14}$. During the same period, a second epidemic took root among intravenous drug users in Yunnan, with strains genetically related to subtype $C$ viruses from India ${ }^{15}$. Co-existence of multiple subtypes led to the formation of circulating recombinant forms (CRFs) of HIV-1 - CRF07_BC and CRF08_BC among intravenous drug users along drug trafficking routes ${ }^{16}$ and CRF01_AE in Chinese sex workers who had worked in Thailand ${ }^{17}$. In the mid1990s, viruses closely related to CRF01_AE and CRF08_BC in Yunnan were identified among intravenous drug users in Guangxi ${ }^{18}$. Further novel recombinants arose in subsequent years ${ }^{19}$.

We compared over 500 nucleotide sequences from Yunnan ${ }^{6,7}$ with those from other provinces in China and neighbouring countries. Comparing sequences from the HIV-1 gag p17 gene with reference sequences from the HIV-1 Database (www.hiv.lanl.gov/ content/index), we identified the three main subtypes of HIV-1 found in Yunnan. These subtypes are those clustering closely with subtype C, CRF07_BC, or CRF08_BC (53.0\%); those with CRF01_ AE or CRF15_01B (40.5\%); and those with subtype B (6.5\%). Notably, more than $90 \%$ of infected intravenous drug users had C/CRF07_BC/CRF08_BC viruses, whereas $85.4 \%$ of CRF01_AE/ CRF15_01B infections were acquired through sexual transmission. Furthermore, sequences in the C/CRF07 BC/CRF08_BC group were found throughout Yunnan, while those in the CRF01_AE/CRF15_01B group were largely confined to prefectures bordering Myanmar. Sequences in the subtype $B$ groups have only been identified in the Dehong and Baoshan prefectures in Yunnan.

The dominant C/CRF07_BC/ CRF08_BC viruses in Yunnan are related to strains in Guangxi province and distant Xinjiang province, supporting the notion that HIV-1 has spread along known drug-trafficking routes. In contrast, sequences similar to CRF01_AE/CRF15_01B have been largely confined to Yunnan and are closely related to strains from Thailand, Myanmar and Vietnam. Subtype B sequences from Yunnan are genetically similar to those from former blood donors in Henan and adjacent provinces, and can be broadly classified into two major groups: one with sequences similar to those from Thailand and Myanmar, and the other with sequences more similar to those in France and the United States. These results are consistent with the hypothesis that HIV-1 spread from Yunnan to central China, and suggest multiple introductions of HIV-1 from foreign countries to Yunnan ${ }^{6}$.

\section{Challenge and opportunity}

Over the past 20 years, HIV-1 in Yunnan has overcome preventive measures to spread beyond high-risk populations. The dramatic increase in sexual transmission has changed the demographic profile of those infected. As the epidemic continues to expand, the genetic makeup of HIV-1 subtypes have become increasingly complex, potentially posing greater challenges to our efforts in antiretroviral treatment and vaccine development.

In light of the observed demographic changes, HIV-1 prevention strategies must focus more on stopping sexual transmission of HIV-1 within high-risk groups and halting the spread to the general public. There are urgent needs to scale up and integrate those proven successful prevention programmes such as condom promotion among female sex workers; drug rehabilitation, needle exchange 
and methadone maintenance for intravenous drug users ; and free antiretroviral therapy for those infected.

There is an old Chinese saying: "When there is a crisis, there is an opportunity." Indeed, as HIV-1 plagues certain high-risk groups in China, there is still a window of opportunity to prevent further spread to the general population. The time to act is now.

1. Wu, Z., Sullivan, S. G., Wang, Y., Rotheram-Borus, M. J. \& Detels, R. Lancet 369, 679-690 (2007).

2. Wu, Z. Y., Liu, Z. Y. \& Detels, R. Lancet 346, 61-62 (1995).

3. Kaufman, J. \& Jing, J. Science 296, 2339-2340 (2002).

4. State Council AIDS Working Committee Office and UN Theme Group on AIDS in China, A Joint Assessment of HIV/ AIDS Prevention, Treatment and Care in China (2007).
5. Gill, B., Huang, Y. \& Lu, X. Demography of HIV/AIDS in China Center for Strategic and International Studies (2007).

6. Zhang, L. et al. J. Virol. 78, 13591-13599 (2004).

7. Zhang, Y. et al. PLoS Med. 3, e443 (2006).

8. US Department of State Bureau for International Narcotics and Law Enforcement Affairs. International Narcotics Control Strategy Report Volume I Drug and Chemical Control (2007).

9. Chin, K.-L. \& Zhang, S. US Department of Justice Report, The Chinese Connection: Cross-border Drug Trafficking between Myanmar and China (2007).

10. Zheng, X. et al. AIDS 8, 1141-1147 (1994)

11. Xiao, Y., Kristensen, S., Sun, J., Lu, L. \& Vermund, S. H. Soc. Sci. Med. 64, 665-675 (2007).

12. Parekh, B. S., Pau, C. P., Kennedy, M. S., Dobbs, T. L. \& McDougal, J. S. AIDS Res. Hum. Retroviruses 17, 137-146 (2001).

13. Graf, M. et al. AIDS Res. Hum. Retroviruses 14, 285-288 (1998).

14. Weniger, B. G., Takebe, Y., Ou, C. Y. \& Yamazaki, S. AIDS
8, S13-S28 (1994)

15. Luo, C. C. et al. Lancet 345, 1051-1052 (1995)

16. Su, L. et al. J. Virol. 74, 11367-11376 (2000).

17. Cheng, H., Zhang, J., Capizzi, J., Young, N. L. \& Mastro, T. D. Lancet 344, 953-954 (1994)

18. Piyasirisilp, S. et al. J. Virol. 74, 11286-11295 (2000).

19. Yang, R. et al. J. Virol. 77, 685-695 (2003).

Acknowledgement This work is supported by National Basic Research Program (also called 973 Program) of Chinese Ministry of Science and Technology to L.Z. (2006CB504200) and by the Tenth Five-year Key Technologies R\&D Programme of China:2004BA719A14-1,2004BA719A14-2. We also thank Mark Goldin for helpful suggestions.

Author information Correspondence should be addressed to L.Z. (e-mail Izhang07@hotmail.com or jiangyan03@263.net).L.L. and M.J.contributed equally to this work.

\section{Authorship}

Lin Lu', Manhong Jia', Yanling Ma', Li Yang ${ }^{1}$, Zhiwei Chen², David D. Ho ${ }^{3,4}$, Yan Jiang ${ }^{5 \star}$ and Linqi Zhang ${ }^{4,6 \star}$

'Yunnan Center for Disease Control and Prevention, Yunnan, People's Republic of China. ${ }^{2}$ AIDS Institute, The University of Hong Kong Li Ka Shing Faculty of Medicine, Hong Kong SAR, People's Republic of China. ${ }^{3}$ Aaron Diamond AIDS Research Center, The Rockefeller University, New York, USA. ${ }^{4}$ Comprehensive AIDS Research Center, Tsinghua University, Beiijing, People's Republic of China. ${ }^{5}$ National AIDS Reference Laboratory, National Center for AIDS/STD Control and Prevention, Chinese Center for Disease Control and Prevention, Beijing, People's Republic of China. ${ }^{6}$ AIDS Research Center, State Key Laboratory for Molecular Virology and Genetic Engineering, Institute of Pathogen Biology, Chinese Academy of Medical Sciences and Peking Union Medical College, Beijing, People's Republic of China. 